\title{
DESIGN OF A MOSFET BASED PORTABLE AND COMPATIBLE DIMMER ADAPTOR FOR LED BULBS
}

\author{
Anubhuti Sharma ${ }^{1}$ and Anshul ${ }^{2}$
}

\begin{abstract}
In a scenario where protection of energy resources and assuring that they remain on the face of earth for a long time, looking for alternatives that support the concept of environment friendly technologies becomes of paramount importance. As we all know that the newer form of technology that is LED is not only famous for its energy savings concept but also for its economic advantage and environment friendly nature over other forms of technology. Because of energy savings and meeting the growing demand for energy production, dimming of LEDs becomes more important to look into. The problem that is being faced when we look into dimming is that the market available dimmers consisting of TRIAC based circuits are not compatible with higher rating LED bulbs that are being used in every household. The major disadvantage of such dimmers include harmonic losses, thereby reducing efficiency of the bulb and finally reducing the life span of the bulb to a considerable amount. Looking into such drawbacks, gave us our main objective that is to come up with a circuit with a newer approach in order to eliminate such losses. The proposed work includes the switching capability of a MOSFET in order to achieve automated turning on and off of the LED lamp. Gate terminal of the MOSFET is triggered by providing the gate pulse through 555 Timer used in a-stable configuration. The desired range of dimming levels are accomplished by altering the turn on and turn off time of the gate pulse which is discussed in detail later. An oscilloscope is used to monitor the waveforms of the gate as well as drain terminal of the MOSFET. Later on, a study has been done as to show which circuit is more efficient, MOSFET based or TRIAC based.
\end{abstract}

Keywords: MOSFET, TRIAC, 555 TIMER.

\section{INTRODUCTION}

Energy efficiency is one of the main highlights when we look into smart lighting. It basically consists of installation of high efficiency luminaires and associated control based on the user's requirement, luminance levels required, type of work area and other factors. When we look into dimming ability of LED Lamps, it brings with it a plethora of advantages which makes this field highly important. Dimming can help increase the lifetime of the LED and that of the associated fixtures to a great extent, almost double it. Not to forget dimming can also decrease the power input thereby reducing energy costs, for instance if fifty percent of the light intensity output is reduced then potentially half of the actual energy required can be saved. So the main objective of our work is to design a MOSFET based circuit in order to achieve a wide dimming range for market available LEDs. The major problem that the already available dimmers is that they are TRIAC based and we know that TRIAC is not able to switch both sides of the AC waveform symmetrically and that leads to high levels of harmonics in the circuit thereby reducing efficiency. Also these dimmers are prone to Electromagnetic interference which further reduces their productivity and use. And all of these drawbacks led us into finding a new circuit based on MOSFET instead of a TRIAC to get rid of harmonics and EMI problem.

The segmentation is as follows. Section II contains the proposed solution and its explanation in detail. Experimental results are discussed in section III. Section IV contains conclusion and future scope of the project.

Lingling Feng et. al [1] talks about the use of an analog based circuit to accomplish dimming. The principle behind this consists of the use of a duty cycle that can be controlled so that the reference voltage is altered which further

${ }^{1}$ Department of Electrical and Electronics Engineering MIT, Manipal, Karnataka, India

${ }^{2}$ Department of Electrical and Electronics Engineering MIT, Manipal, Karnataka, India 
decides the duty cycle of pulse modulated wave and that later on changes the current given to LED. This is how dimming is done by basically changing the LED output current. Ruihong Zhang et. al [2] gives a solution regarding how to increase the dimming range of LED lamps. The concept is based on making the value of input current provided to LED greater than the TRIAC's holding current by altering the input reactive power provided to the LED. The circuitry consists of a 4 quadrant ac-dc converter in order to shape the input current and a resonant converter for regulation of reactive power. The switching circuit for the two converters mentioned above is the same. Hussain A. Attia et. al [3] has devised a decentralized control street lighting system that works based on the conditions like luminance levels required on the road, amount of traffic on the road, daylight conditions et cetera. The conventional street lighting has centralized control which exercises dimming based on how fast the data is transferred from the street light to the control room, hence it fails to be more reliable and fast. In order to eliminate this, the author talks about having a decentralized control mounted on the street light pole itself so that light shifts due to traffic conditions and light level requirements are more reliable and fast. E. S. Lee et. a1 [4] Here the author's work is based on the concept of triode based dimming. The circuitry a variable switched capacitor in order to control the dimming of the LED and also a variable resistor pot is used to control turning on and off of the triode switch. The advantages of the circuit involves reliability and longer life span. S. Moon [5] has designed a model based on the concept of a feedback control dimmer LED driver. It works on the principle of controlling the LED output current in accordance with the phase angle of the TRIAC switch used in the LED driver circuit. Xiaoru Xu et. a1 [6] Here the author talks about a higher percentage LED driver that consists of a power boost stage. The advantage of this solution is that it eliminates harmonics. W. Ma et. a1 [7] talks about using an adaptive bleeder circuitry for regulation of bleeder current in accordance with phase angle to reduce harmonics. The driver works around boundary conduction model in larger phase angle for accomplishing higher efficiency. Wider dimming angle can be achieved using a non-linear current reference.

\section{Proposed Algorithm}

\section{Hardware and working Analysis:}

The proposed solution presents the concept of using a MOSFET based switching network that is able to control the automated turning on and off of the LED lamp. In order to trigger the MOSFET, a gate pulse is required that is able to shift the MOSFET from its cut-off region to its active region. This is done by providing the gate pulse through the output pin of a 555 Timer used in astable multivibrator configuration. The concept of dimming in this solution includes that if we are able to change the width of the pulse being provided to the MOSFET, the apparent luminance levels of the LED lamp can be simultaneously varied. Basically it depends on how well the person's eye can integrate the average luminance output given by the LED bulb or in other words " persistence of vision".

Now, a further insight into the circuitry involved. To start with, an auto transformer of rating 0-300 volts is being used in order to feed the circuit with AC supply. Since the LED works on DC supply, we have used a bridge rectifier for conversion of $\mathrm{AC}$ into pulsed DC. The rectifier has two ends, one being the higher end and other being the lower end. The high or positive end is joined at the first terminal of LED lamp and the low or negative end to sourc of the MOSFET. Drain of MOSFET is joined to the other terminal of LED lamp. Gate is being triggered by the pulse provided by pin three of 555 Timer. Timer uses its own DC supply which is kept constant at 5 volts. The pin diagram of the timer circuit is given below:

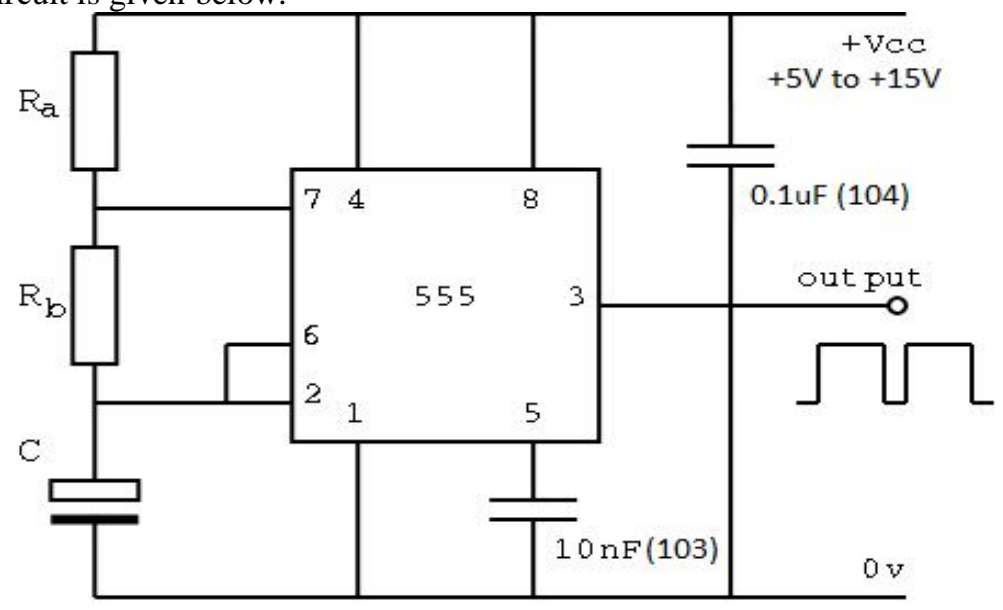

Figure 1. Timer circuit pin connection diagram 


\section{Working:}

First, the AC supply is fed to the circuit and is kept between 140-150 volts. In the circuit, Rb is kept variable by using a variable resistance pot. $\mathrm{Ra}$ is kept constant. By varying the value of $\mathrm{Rb}$, the width of the gate pulse simultaneously varies. A wider pulse gives brighter light and a narrow pulse gives lesser luminance level. Hence, by varying the duty ratio, light intensity of the LED bulb can be automated. The switching circuit consists of the MOSFET. This type of switching is symmetrical and therefore does not involve harmonics. It is an efficient technique and such a circuit provides good color rendering index.

Mathematical Equations applied for accomplishing required time delay:

Turn on time $=0.693 *(\mathrm{Ra}+\mathrm{Rb}) * \mathrm{C}$

Turn off time $=0.693 * \mathrm{Rb} * \mathrm{C}$

Sample Calculation: If $\mathrm{Ra}=3.2$ Kilo ohms, $\mathrm{Rb}=4.88$ Kilo ohms and $\mathrm{C}=0.1$ Micro farad, then Turn on time $=0.693$ $((3.2+4.88) * 103)(0.1 * 10-6)=559.4$ milli-seconds, Turn off time $=0.693(4.88 * 103)(0.1 * 10-6)=338$ milli seconds.

Hence, duty cycle $=62 \%$

The value of resistor may vary in the future because we are working on getting a lower delay.

\section{The final circuit diagram:}

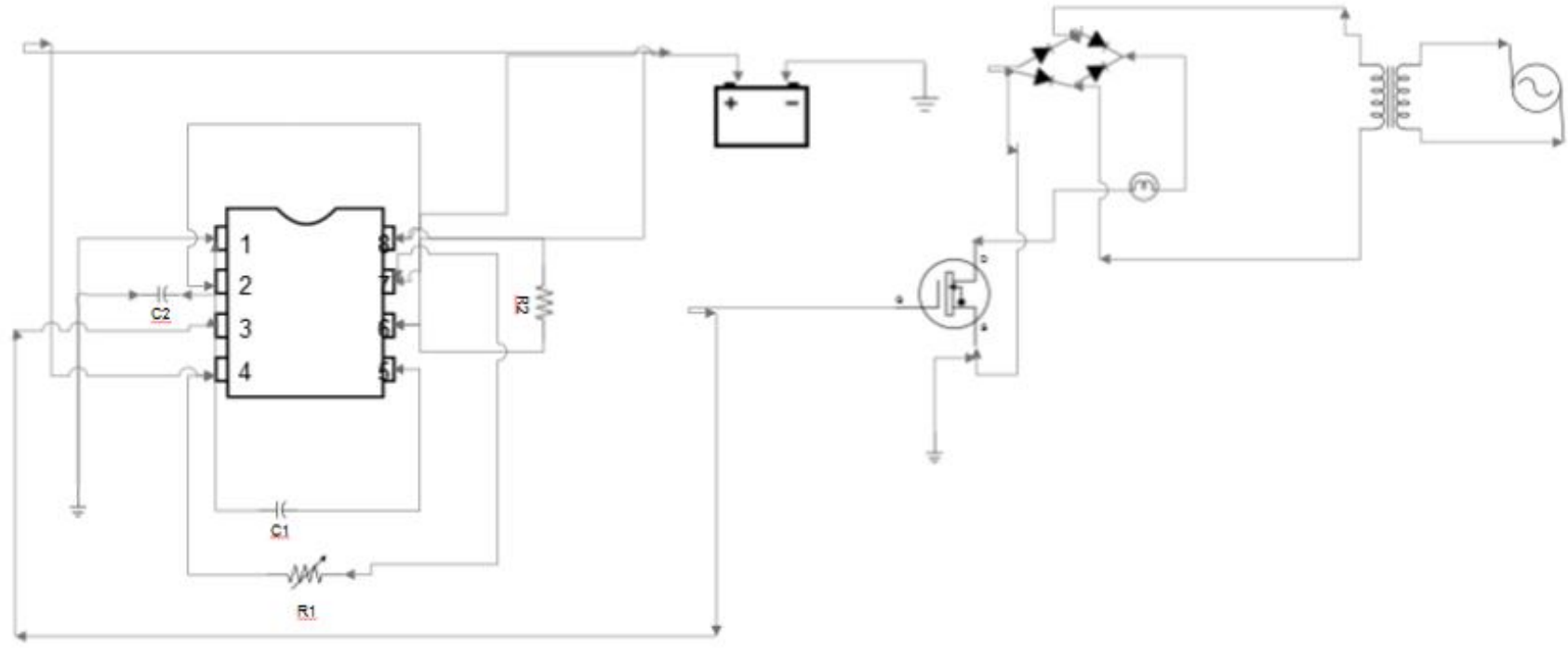

Figure 2. Circuit diagram of dimmer adaptor for LED Lamps

As depicted in the picture, The MOSFET is triggered by the timer pulse at gate terminal. The dimming can be visually seen and the values of luminance levels can be recorded using a lux meter in a dark room.

\section{EXPERIMENT AND RESULT}

The AC supply is switched on as shown in the circuit diagram above, LED glows brightly. An oscilloscope is used in order to monitor the waveforms. Channel 1 is connected to the gate terminal of the MOSFET and channel 2 is connected to the drain terminal of the MOSFET. At this point, we start varying the resistance value through variable resistance pot. As a result the gate pulse varies which is visible in channel 1, simultaneously we are getting a complementary waveform in channel 2 connected to the drain. The complementary nature of the waveforms show that the MOSFET is working as a switch and not going into saturation. As the resistance value varies, brightness varies. Increasing resistance, increases turn on time with turn off time being constant and simultaneously brightness increases. Decreasing resistance decreases turn on time and brightness decreases. In this way, at different values of resistance, different dimming levels are achieved. 
The light intensity at different values of resistance can be measured by a lux meter by pointing at the middle of the LED lamp from a large distance (preferably thrice the radius of the lamp) in a black room. The light intensity is compared against the light intensity provided by the lamp when used independently and hence percentage dimming is calculated. As of now, 30-100 \% dimming has been achieved but in future we will be trying to make it 10-100\%.

IMPLEMENTATION:

The above proposal has been implemented on a market available LED lamp with rating of 15 Watts. The illuminance levels are recorded using a lux meter by pointing at the middle of the LED lamp from a large distance (preferably thrice the radius of the lamp). The table below gives the recorded values. These values were recorded at DC end and in the absence of a black room so these are relative values that include the indirect component of light as well:

Illuminance of the bulb without using the circuit is 2250 lumens

Table -1 Experiment Result

\begin{tabular}{|l|l|l|l|}
\hline Serial No. & Resistance Value & Illuminance Level & \%Dimming \\
\hline 1 & $100 \mathrm{k}$ & 2005 & 10.89 \\
\hline 2 & $70 \mathrm{k}$ & 1523 & 32.31 \\
\hline 3 & $50 \mathrm{k}$ & 1116 & 50.40 \\
\hline 4 & $30 \mathrm{k}$ & 556 & 75.28 \\
\hline
\end{tabular}

The resistance values are achieved by varying the resistance pot. The resistance pot used here is rated from $0-100 \mathrm{k}$ ohms. Different values of illuminance can be achieved by varying the resistance value, for now we have taken four values for reference. The theory behind this implementation is mentioned above. As we can see, dimming between $10-75 \%$ has been achieved and further it can be made more flexible by using precise values of resistance from the pot.

\section{Comparison:}

The following table depicts the major differences between TRIAC based Dimming and MOSFET based Dimming. It clearly mentions the disadvantages of TRIAC and how MOSFET is a better option that can be utilized to achieve desired range of dimming.

\begin{tabular}{|l|l|}
\hline \multicolumn{1}{|c|}{ TRIAC Dimming } & \multicolumn{1}{|c|}{ MOSFET Dimming } \\
\hline Non-symmetrical firing on both sides of AC waveform. & Symmetrical firing on both sides of AC waveform. \\
\hline $\begin{array}{l}\text { Non-symmetrical firing leads to high level harmonics } \\
\text { thereby reducing efficiency. }\end{array}$ & Highly efficient as it is not susceptible to harmonics. \\
\hline $\begin{array}{l}\text { Electromagnetic Interference problem is faced due to non- } \\
\text { symmetrical switching. }\end{array}$ & No such problem. \\
\hline $\begin{array}{l}\text { 10-50 \% dimming levels are achieved. } \\
\text { A typical market available TRIAC based dimmer circuit } \\
\text { ranges between 3-5\$ }\end{array}$ & $\begin{array}{l}\text { The circuit that we have come up with ranges between 1- } \\
3 \$\end{array}$ \\
\hline
\end{tabular}




\section{IV.CONCLUSION}

A circuit has been designed using MOSFET as a switch and 555 Timer as the gate pulse provider. This circuit is used to achieve different light intensities of a LED bulb by varying the duty cycle of the gate pulse. The main highlight is that it can be used on any rating LED lamp. When we look into its economical nature, since it is a more efficient driver than a TRIAC based circuit and does not cause any harmonics, it increases the lifetime of LEDS thereby reducing installation and maintenance costs. The dimming range given by this circuit is also found out to be wide and flexible. Desired illuminance levels can be set by using precise values of resistance from the pot. Another advantage of this circuit is that it is being modulated at the AC end that is the supply side, therefore on the DC end we can use any rating LED lamp. Also incandescent lamp can be used instead of LED bulb, in that case we can utilize its dimming property to get different thermal levels of the filament inside it and can be used as a mini thermal chamber.

\section{REFERENCES}

[1] Lingling Feng, Yingna Zhou, Yong Peng, and Wei Li. A new analog dimming circuit used in led driver. In Solid-State and Integrated Circuit Technology (ICSICT), 2014 12th IEEE International Conference on, pages 1-3. IEEE, 2014.

[2] Ruihong Zhang and Henry Shu-hung Chung. A triac-dimmable led lamp driver with wide dimming range. IEEE Transactions on Power Electronics, 29(3):1434- 1446, 2014.

[3] H. A. Attia, A. Omar and M. Takruri, "Design of decentralized street LED light dimming system," 2016 5th International Conference on Electronic Devices, Systems and Applications (ICEDSA), Ras Al Khaimah, 2016

[4] E. S. Lee, B. H. Choi, D. T. Nguyen and C. T. Rim, "The analysis of TRIAC dimming LED driver by variable switched capacitor for long life and high power-efficient applications," 2015 9th International Conference on Power Electronics and ECCE Asia (ICPE-ECCE Asia), Seoul, 2015

[5] S. Moon, G. B. Koo and G. W. Moon, "Dimming-Feedback Control Method for TRIAC Dimmable LED Drivers," in IEEE Transactions on Industrial Electronics, vol. 62, no. 2, pp. 960-965, Feb. 2015.

[6] Xiaoru Xu and Xiaobo Wu, "High dimming ratio LED driver with fast transient boost converter," 2008 IEEE Power Electronics Specialists Conference, Rhodes, 2008

[7] W. Ma, X. Xie, Y. Han and H. Deng, "Control scheme for TRIAC dimming high PF single-stage LED driver with adaptive bleeder circuit and non-linear current reference," 2016 IEEE Applied Power Electronics Conference and Exposition (APEC), Long Beach, CA, 2016 